\title{
On the Main Signless Laplacian Eigenvalues of a Graph *
}

\author{
Hanyuan Deng; He Huang \\ College of Mathematics and Computer Science, \\ Hunan Normal University, Changsha, Hunan 410081, P. R. China
}

2011-7-1

\begin{abstract}
A signless Laplacian eigenvalue of a graph $G$ is called a main signless Laplacian eigenvalue if it has an eigenvector the sum of whose entries is not equal to zero. In this paper, we first give the necessary and sufficient conditions for a graph with one main signless Laplacian eigenvalue or two main signless Laplacian eigenvalues, and then characterize the trees and unicyclic graphs with exactly two main signless Laplacian eigenvalues, respectively.
\end{abstract}

Keywords: Signless Laplacian eigenvalue; main eigenvalue; tree; unicyclic graph.

\section{Introduction}

Let $M$ be a square matrix of order $n$, an eigenvalue $\lambda$ of $M$ is said to be a main eigenvalue if the eigenspace $\varepsilon(\lambda)$ of $\lambda$ is not orthogonal to the all-1 vector $\mathbf{j}$, i.e., it has an eigenvector the sum of whose entries is not equal to zero. An eigenvector $\mathbf{x}$ is a main eigenvector if $\mathbf{x}^{\mathbf{T}} \mathbf{j} \neq 0$. Specially, if $M=A$ is the $(0,1)$-adjacency matrix of a graph $G$, then the main eigenvalues of $A$ are

\footnotetext{
${ }^{*}$ This work was supported by Hunan Provincial Natural Science Foundation of China (09JJ6009) and the Program for Science and Technology Innovative Research Team in Higher Educational Institution of Hunan Province.

${ }^{\dagger}$ Corresponding author: hydeng@hunnu.edu.cn.
} 
said to be main eigenvalues of $G$. A graph with exactly one main eigenvalue is regular. Cvetković [1] proposed the problem of characterizing graphs with exactly $\mathrm{k}$ main eigenvalues, $k>1$. Hagos [3] gave a characterization of graphs with exactly two main eigenvalues. Recently, Hou and Zhou [4] characterized the tree with exactly two main eigenvalues. Hou and Tian [5] determined all connected unicyclic graphs with exactly two main eigenvalues. Zhu and $\mathrm{Hu}$ [6] characterized all connected bicyclic graphs with exactly two main eigenvalues. Rowlinson [7] surveyed results relating main eigenvalues and main angles to the structure of a graph, and discussed graphs with just two main eigenvalues in the context of measures of irregularity and in the context of harmonic graphs.

In this paper, we assume that $G$ is a simple connected graph, and will consider the main eigenvalues of the signless Laplacian matrix $Q$ of $G$, where $Q=D+A$ and $D$ is the diagonal matrix of vertex degrees. The main eigenvalues of $Q$ is said to be the main signless Laplacian eigenvalues of $G$. The signless Laplacian appears very rarely in published papers before 2003. Recently the signless Laplacian has attracted the attention of researchers, see, e.g. [8] [9] [10] [11] [12]. Here, we will give the necessary and sufficient conditions for a graph with one main signless Laplacian eigenvalue or two main signless Laplacian eigenvalues, and then characterize the trees and unicyclic graphs with exactly two main signless Laplacian eigenvalues, respectively.

\section{The graphs with one or two main signless Laplacian eigenvalues}

In this section, we will show that a graph with exactly one main signless Laplacian eigenvalue is regular, and give a characterization of graphs with exactly two main signless Laplacian eigenvalues. 
Note that if $G$ is a simple connected graph with signless Laplacian matrix $Q$, then there is an eigenvector $\mathbf{x}>0$ of the largest eigenvalue $\mu_{1}$ of $Q$ such that $Q \mathbf{x}=\mu_{1} \mathbf{x}$, and $x^{T} \mathbf{j} \neq 0$ by the Perron-Frobenius theorem. This shows that the largest eigenvalue $\mu_{1}$ of $Q$ is a main signless Laplacian eigenvalue. So, $G$ has at least one main signless Laplacian eigenvalue.

The following result gives a characterization of graphs with exactly one main signless Laplacian eigenvalue.

Theorem 1. A graph $G$ with exactly one main signless Laplacian eigenvalue if and only if $G$ is regular.

Proof. If $G$ is $k$-regular, then $Q \mathbf{j}=2 k \mathbf{j}$. This shows that $\mu_{1}=2 k$ is an eigenvalue of $Q$ with an eigenvector $\mathbf{j}$. Since $Q$ is a non-negative irreducible symmetric matrix, $\mu_{1}=2 k$ is the largest eigenvalue of $Q$ with the multiplicity 1 by the Perron-Frobenius theorem. And the eigenvectors of other eigenvalues of $Q$ are orthogonal with $\mathbf{j}$. So, $Q$ has exactly one main eigenvalue.

If $G$ has exactly one main signless Laplacian eigenvalue, then the largest eigenvalue $\mu_{1}$ is the unique main eigenvalue of $Q$. Let $\xi$ be a eigenvector of $\mu_{1}, \mathbf{V}_{\mathbf{1}}=\varepsilon\left(\mu_{1}\right)$ the eigenspace of $\mu_{1}$, then $\mathbf{V}_{\mathbf{1}}$ is the space spanning by $\xi$. If $\mathbf{V}_{\mathbf{2}}$ is the space spanning by eigenvectors of all eigenvalues of $Q$ different from $\mu_{1}$, and $\mathbf{V}_{\mathbf{3}}$ is the space spanning by $\mathbf{j}$, then $\operatorname{dim}\left(\mathbf{V}_{\mathbf{2}}\right)=n-1$ and $\operatorname{dim}\left(\mathbf{V}_{\mathbf{3}}\right)=1$. Since $Q$ is a real symmetric matrix, $\mathbf{V}_{\mathbf{1}}$ is the orthogonal complement of $\mathbf{V}_{\mathbf{2}}$. And $\mathbf{V}_{\mathbf{3}}$ is also the orthogonal complement of $\mathbf{V}_{\mathbf{2}}$ since $\mu_{1}$ is the unique main eigenvalue of $Q$. So, $\mathbf{V}_{\mathbf{1}}=\mathbf{V}_{\mathbf{3}}$, and $\xi=a \mathbf{j}$ for some real $a \neq 0$. From $Q \xi=\mu_{1} \xi$, the row sums of $Q$ are equal, and $G$ is regular.

Now, we discuss the characterization of graphs with exactly two main signless Laplacian eigenvalues.

For any positive semi-definite matrix $M$ of order $n$, all its eigenvalues 
are non-negative. Let $\mu_{1}>\mu_{2}>\cdots>\mu_{r}$ be the eigenvalues of $M$ with multiplicities $n_{1}, n_{2}, \cdots, n_{r}$, respectively, where $n_{1}+n_{2}+\cdots+n_{r}=n$. $\left\{\xi_{i 1}, \xi_{i 2}, \cdots, \xi_{i n_{i}}\right\}$ is a standard and orthogonal basis of the eigenspace $\varepsilon\left(\mu_{i}\right)$, $i=1,2, \cdots, r$. And $P=\left[\xi_{11}, \cdots, \xi_{1 n_{1}}, \xi_{21}, \cdots, \xi_{2 n_{2}}, \cdots, \xi_{r 1}, \cdots, \xi_{r n_{r}}\right], P_{i}=$ $\left[0, \ldots, \xi_{i 1}, \ldots, \xi_{i n_{i}}, 0, \ldots, 0\right], \mathbf{j}=[1,1, \ldots, 1]^{T}$, then

$$
P^{T} M P=\left(\begin{array}{cccc}
\mu_{1} I_{n_{1} \times n_{1}} & 0 & \ldots & 0 \\
0 & \mu_{2} I_{n_{2} \times n_{2}} & \ldots & 0 \\
\vdots & \vdots & \ddots & \vdots \\
0 & 0 & \ldots & \mu_{r} I_{n_{r} \times n_{r}}
\end{array}\right)
$$

Let

$$
E_{i}=\left(\begin{array}{ccccc}
0 & \ldots & \ldots & \ldots & 0 \\
\vdots & \ddots & \vdots & \vdots & \vdots \\
\vdots & \vdots & I_{n_{i} \times n_{i}} & \vdots & \vdots \\
\vdots & \vdots & \vdots & \ddots & \vdots \\
0 & \ldots & \ldots & \ldots & 0
\end{array}\right)
$$

$Q_{i}=P E_{i} P^{T}$, then

$$
Q_{i} \mathbf{j}=P E_{i} P^{T} \mathbf{j}=P_{i} P_{i}^{T} \mathbf{j}
$$

and $M$ has the spectral decomposition

$$
M=\mu_{1} Q_{1}+\mu_{2} Q_{2}+\cdots+\mu_{r} Q_{r}
$$

where

$$
Q_{i} Q_{j}= \begin{cases}\mathbf{0} & i \neq j \\ Q_{i} & i=j\end{cases}
$$

And, for any polynomial $f(x)=a_{0} x^{n}+a_{1} x^{n-1}+\cdots+a_{n-1} x+a_{n}$,

$$
\begin{aligned}
f(M) & =a_{0}(M)^{n}+a_{1}(M)^{n-1}+\ldots+a_{n-1} M+a_{n} I \\
& =a_{0} \sum_{i=1}^{r} \mu_{i}^{n} Q_{i}+a_{1} \sum_{i=1}^{r} \mu_{i}^{n-1} Q_{i}+\cdots+a_{n-1} \sum_{i=1}^{r} \mu_{i} Q_{i}+a_{n} \sum_{i=1}^{r} Q_{i} \\
& =\sum_{i=1}^{r} f\left(\mu_{i}\right) Q_{i}
\end{aligned}
$$


Lemma 2. Let $\mu_{1}, \mu_{2}, \ldots, \mu_{t}(1 \leq t \leq r)$ be the main eigenvalues of a positive semi-definite matrix $M$ of order $n$, and $m(x)=\left(x-\mu_{1}\right)(x-$ $\left.\mu_{2}\right) \cdots\left(x-\mu_{t}\right)$, then

(i) $m(M) \mathbf{j}=0$;

(ii) If $f(x)$ is a polynomial with real coefficients and $f(M) \mathbf{j}=0$, then $m(x) \mid f(x)$.

And

Proof. (i) From (2), we know that $m(M)=\sum_{i=1}^{r} m\left(\mu_{i}\right) Q_{i}=\sum_{i=t+1}^{r} m\left(\mu_{i}\right) Q_{i}$.

$$
m(M) \mathbf{j}=\sum_{i=t+1}^{r} m\left(\mu_{i}\right) Q_{i} \mathbf{j}
$$

Since $\mu_{t+1}, \cdots, \mu_{r}$ are not the main eigenvalues of $M$, and from (1),$Q_{i} \mathbf{j}=$ 0 for $i=t+1, \cdots, r$. So, $m(M) \mathbf{j}=\sum_{i=t+1}^{r} m\left(\mu_{i}\right) Q_{i} \mathbf{j}=0$.

(ii) From (2) and $Q_{i} \mathbf{j}=0$ for $i=t+1, \cdots, r$,

$$
f(M) \mathbf{j}=\sum_{i=1}^{r} f\left(\mu_{i}\right) Q_{i} \mathbf{j}=\sum_{i=1}^{t} f\left(\mu_{i}\right) Q_{i} \mathbf{j} .
$$

Since $f(M) \mathbf{j}=0, \sum_{i=1}^{t} f\left(\mu_{i}\right) Q_{i} \mathbf{j}=0$. For $k=1,2, \ldots, t$, we have $Q_{k}\left(\sum_{i=1}^{t} f\left(\mu_{i}\right) Q_{i} \mathbf{j}\right)=$ $f\left(\mu_{k}\right) Q_{k} \mathbf{j}=0$. So, $f\left(\mu_{k}\right)=0$ for $k=1,2, \ldots, t$ and $m(x) \mid f(x)$.

A number $\alpha$ is an algebraic integer if there is a monic polynomial $f(x)$ with integral coefficients such that $f(\alpha)=0$.

Lemma 3( [13]). $\alpha \in \mathbb{Q}$ is an algebraic integer if and only if $\alpha$ is an integer.

Lemma 4([13]). If $\alpha$ and $\beta$ are algebraic integers, then $\alpha \pm \beta$ and $\alpha \beta$ are also algebraic integers.

Theorem 5. Let $G$ be non-regular, then $G$ has exactly two main signless Laplacian eigenvalues $\mu_{1}$ and $\mu_{2}$ if and only if $\left(Q-\mu_{1} I\right)\left(Q-\mu_{2} I\right) \mathbf{j}=0$.

Proof. Let $\mu_{1}, \ldots, \mu_{t}$ be the main eigenvalues of $Q$, and $m(x)=(x-$ $\left.\mu_{1}\right) \cdots\left(x-\mu_{t}\right)$. 
If $\left(Q-\mu_{1} I\right)\left(Q-\mu_{2} I\right) \mathbf{j}=0$, then $f(Q) \mathbf{j}=0$ for $f(x)=\left(x-\mu_{1}\right)\left(x-\mu_{2}\right)$, and $m(x) \mid f(x)$ by Lemma 2. So, $m(x)=\left(x-\mu_{1}\right)\left(x-\mu_{2}\right)$ or $\left(x-\mu_{1}\right)$ or $\left(x-\mu_{2}\right)$, and $t \leq 2$. But $G$ is non-regular, $t=2$ from Theorem 1 .

If $G$ has exactly two main signless Laplacian eigenvalues $\mu_{1}$ and $\mu_{2}$, then $\left(Q-\mu_{1} I\right)\left(Q-\mu_{2} I\right) \mathbf{j}=0$ from Lemma 2.

In the following, we give an alternative characterization of graphs with exactly two main signless Laplacian eigenvalues.

In order to find all graphs with exactly two main eigenvalues, Hou and Tian [5] introduced a 2-walk $(a, b)$-linear graph. For a graph $G$, the degree of vertex $v$ is denoted by $d(v)$, the number of walks of length 2 of $G$ starting at $v$ is $s(v)=\sum_{u \in N_{G}(v)} d(u)$, i.e., the sum of the degrees of the vertices adjacent to $v$, where $N_{G}(v)$ is the set of all neighbors of $v$ in $G$. A graph $G$ is called 2 -walk $(a, b)$-linear if there exist unique integer numbers $a, b$ with $a^{2}-4 b>0$ such that $s(v)=a d(v)+b$ holds for every vertex $v \in V(G)$. Hagos [3] showed that a graph $G$ has exactly two main eigenvalues if and only if $G$ is 2 -walk linear.

Like a 2-walk $(a, b)$-linear graph, we definite a 2-walk $(a, b)$-parabolic graph. A graph $G$ is called 2-walk $(a, b)$-parabolic if there are uniquely a positive integer $a$ and a non-negative integer $b$ with $a^{2}-8 b>0$ such that $s(v)=-d^{2}(v)+a d(v)-b$ holds for every vertex $v \in V(G)$.

Theorem 6. A graph $G$ has exactly two main signless Laplacian eigenvalues if and only if $G$ is a 2 -walk $(a, b)$-parabolic graph.

Proof. If $G$ is a 2-walk $(a, b)$-parabolic graph, then there are uniquely a positive integer $a$ and a non-negative integer $b$ such that $a^{2}-8 b>0$ and $s(v)=-d^{2}(v)+a d(v)-b$ for any $v \in V(G)=\left\{v_{1}, v_{2}, \cdots, v_{n}\right\}$. So, 
$s\left(v_{i}\right)+d^{2}\left(v_{i}\right)-a d\left(v_{i}\right)+b=0$, and

$$
\begin{aligned}
\frac{1}{2}(A+D)^{2} \mathbf{j}-a A \mathbf{j}+b \mathbf{j} & =\mathbf{0} \\
\frac{1}{2} Q^{2} \mathbf{j}-\frac{1}{2} a L^{+} \mathbf{j}+b \mathbf{j} & =\mathbf{0} \\
Q^{2} \mathbf{j}-a Q \mathbf{j}+2 b \mathbf{j} & =\mathbf{0}
\end{aligned}
$$

Let $f(x)=x^{2}-a x+2 b$, then $f(Q) \mathbf{j}=\mathbf{0}$, and $f(x)=0$ has two real roots since $a^{2}-8 b>0$. And $G$ is non-regular since one has $s(v)=-d^{2}(v)+$ $2 k d(v)-0$ and $s(v)=-d^{2}(v)+(2 k+1) d(v)-k$ for a $k$-regular graph, i.e., $(a, b)=(2 k, 0)$ or $(2 k+1, k)$ is not unique. From Theorem $5, G$ has exactly two main signless Laplacian eigenvalues.

On the other hand, if $G$ has exactly two main signless Laplacian eigenvalues $\mu_{1}$ and $\mu_{2}$, then by Theorem 5 ,

$$
\left(Q^{2}-\left(\mu_{1}+\mu_{2}\right) Q+\mu_{1} \mu_{2} I\right) \mathbf{j}=\mathbf{0}
$$

i.e.,

$$
(D+A)^{2} \mathbf{j}-\left(\mu_{1}+\mu_{2}\right)(D+A) \mathbf{j}+\mu_{1} \mu_{2} \mathbf{j}=\mathbf{0} .
$$

So, $d^{2}(v)+s(v)-\left(\mu_{1}+\mu_{2}\right) d(v)+\frac{\mu_{1} \mu_{2}}{2}=0$ for all $v \in V(G)$. Let $\mu_{1}+\mu_{2}=a$ and $\mu_{1} \mu_{2}=2 b$, then $s(v)=-d^{2}(v)+a d(v)-b$, and $a>0, b \geq 0$ and $a^{2}-8 b>0$ since $\mu_{1} \neq \mu_{2}$ are the eigenvalues of the positive semi-definite matrix $Q$. Note that $G$ is non-regular by Theorem 1 , there are $u, v \in V(G)$ such that $d(u) \neq d(v)$. From $s(u)=-d^{2}(u)+a d(u)-b$ and $s(v)=-d^{2}(v)+a d(v)-b$, we have

$$
\begin{gathered}
a=\frac{s(u)-s(v)}{d(u)-d(v)}+d(u)+d(v) \\
b=\frac{s(u)-s(v)}{d(u)-d(v)} d(v)+d(u) d(v)-s(v)
\end{gathered}
$$

and $a, b$ are rational numbers and unique. Because $\mu_{1}, \mu_{2}$ are the roots of monic polynomial $\operatorname{det}(\lambda I-Q)=0$ with integral coefficients, $\mu_{1}, \mu_{2}$ are algebraic integers. By Lemmas 3 and $4, a, b$ are integers. 


\section{Trees with exactly two main signless Lapla- cian eigenvalues}

In this section, we will determine all trees with exactly two main signless Laplacian eigenvalues.

Let $G=(V, E)$ be a tree with $n \geq 3$ vertices and the maximum degree $\Delta$. If $G$ has exactly two main signless Laplacian eigenvalues, then from Theorem 6 , there exist uniquely a positive integer $a$ and a non-negative integer $b$ such that such that $a^{2}-8 b>0$ and

$$
s(v)=-d^{2}(v)+a d(v)-b
$$

for any $v \in V(G)$.

Case 1. $b=0$.

Let $v_{1} \in V$ with degree $d\left(v_{1}\right)=1$, and $v_{2}$ is its unique adjacent vertex. Then $d\left(v_{2}\right)=s\left(v_{1}\right)=-1+a$ by (41), and

$$
a=d\left(v_{2}\right)+1 \leq \Delta+1
$$

Let $v_{0} \in V$ with degree $d\left(v_{0}\right)=\Delta$, then $\Delta=d\left(v_{0}\right) \leq s\left(v_{0}\right)=-d^{2}\left(v_{0}\right)+$ $a d\left(v_{0}\right)=-\Delta^{2}+a \Delta$, and $\Delta \leq a-1$, i.e.,

$$
a \geq \Delta+1
$$

with equality if and only if $G$ is a star with the center $v_{0}$. From (5) and (6), we have $a=\Delta+1$. So, $G=S_{n}$ is a star.

Case 2. $b=1$.

Let $P_{k}=v_{1} v_{2} \ldots v_{k}$ is a longest path of $G$. Then $d\left(v_{2}\right)=s\left(v_{1}\right)=-1+$ $a-1=a-2$ by (41), and

$$
\begin{aligned}
s\left(v_{2}\right) & =-d^{2}\left(v_{2}\right)+a d\left(v_{2}\right)-1 \\
& =-(a-2)^{2}+a(a-2)-1 \\
& =2 a-5
\end{aligned}
$$


Since $P_{k}=v_{1} v_{2} \ldots v_{k}$ is a longest path of $G$, the adjacent vertices of $v_{2}$ are pendant vertices except $v_{3}$. So, $s\left(v_{2}\right)=\sum_{v \in N_{G}\left(v_{2}\right)} d(v)=d\left(v_{3}\right)+d\left(v_{2}\right)-1$ and

$$
\begin{aligned}
d\left(v_{3}\right) & =s\left(v_{2}\right)-d\left(v_{2}\right)+1 \\
& =(2 a-5)-(a-2)+1 \\
& =a-2=d\left(v_{2}\right)
\end{aligned}
$$

By (4), we have $s\left(v_{3}\right)=s\left(v_{2}\right)=2 a-5=d\left(v_{2}\right)+d\left(v_{3}\right)-1$. This shows that the adjacent vertices of $v_{3}$ are pendant vertices except $v_{2}$. So, $G=S_{\frac{n}{2}, \frac{n}{2}}$ is a double star.

Case 3. $b \geq 2$.

Let $P_{k}=v_{1} v_{2} \ldots v_{k}$ is a longest path of $G$, then $d\left(v_{2}\right) \geq 2$. By (4), $d\left(v_{2}\right)=s\left(v_{1}\right)=-1+a-b$, and $a-b \geq 3$.

Since $P_{k}=v_{1} v_{2} \ldots v_{k}$ is a longest path of $G$, the adjacent vertices of $v_{2}$ are pendant vertices except $v_{3}$.

$$
\begin{aligned}
d\left(v_{3}\right)= & s\left(v_{2}\right)-\left(d\left(v_{2}\right)-1\right) \\
= & \left(-(a-b-1)^{2}+a(a-b-1)-b\right)-\left(d\left(v_{2}\right)-1\right) \\
= & \left(-(a-b-1)^{2}+a(a-b-1)-b\right)-(a-b-2) \\
= & a b-b^{2}-2 b+1 \\
\text { and } \quad d\left(v_{3}\right)-d\left(v_{2}\right) & =\left(a b-b^{2}-2 b+1\right)-(a-b-1) \\
& =a b-b^{2}-b-a+2 \\
& =(b-1)(a-b-2)>0
\end{aligned}
$$

So, $d\left(v_{3}\right)>d\left(v_{2}\right)=a-b-1 \geq 2$. And no pendant vertex is adjacent to $v_{3}$; Otherwise, let $u$ be a pendant vertex adjacent to $v_{3}$. Then $d\left(v_{3}\right)=s(u)=$ $-1+a-b$ by (44), contradicting with $d\left(v_{3}\right)>a-b-1$. 
For any $x \in N_{G}\left(v_{3}\right) \backslash\left\{v_{2}, v_{4}\right\}$, since $x$ is not a pendant vertex, there is $y \in V(G) \backslash\left\{v_{3}\right\}$ such that $x y \in E(G)$, and $y$ is a pendant vertex by the longest path $P_{k}=v_{1} v_{2} \ldots v_{k}$. Then

$$
d(x)=s(y)=-1+a-b=d\left(v_{2}\right), \quad \forall x \in N_{G}\left(v_{3}\right) \backslash\left\{v_{2}, v_{4}\right\}
$$

So,

$$
\begin{aligned}
s\left(v_{3}\right)=\sum_{z \in N_{G}\left(v_{3}\right)} d(z) & =\sum_{x \in N_{G}\left(v_{3}\right) \backslash\left\{v_{2}, v_{4}\right\}} d(x)+d\left(v_{2}\right)+d\left(v_{4}\right) \\
& =\left(d\left(v_{3}\right)-2\right) d\left(v_{2}\right)+d\left(v_{2}\right)+d\left(v_{4}\right)
\end{aligned}
$$

and $\quad d\left(v_{4}\right)=s\left(v_{3}\right)-\left(d\left(v_{3}\right)-1\right) d\left(v_{2}\right)$

By (4), $s\left(v_{3}\right)=-d^{2}\left(v_{3}\right)+a d\left(v_{3}\right)-b$. Note that $d\left(v_{2}\right)=a-b-1$ and $d\left(v_{3}\right)=a b-b^{2}-2 b+1$,

$$
\begin{aligned}
d\left(v_{4}\right) & =-d^{2}\left(v_{3}\right)+a d\left(v_{3}\right)-b-\left(d\left(v_{3}\right)-1\right) d\left(v_{2}\right) \\
& =d\left(v_{3}\right)\left(-d\left(v_{3}\right)+a-d\left(v_{2}\right)\right)-b+d\left(v_{2}\right) \\
& =d\left(v_{3}\right)\left(-a b+b^{2}+3 b\right)+a-2 b-1 \\
& =d\left(v_{3}\right) b(b-a+3)+(a-2 b-1)
\end{aligned}
$$

If $a-b=3$, then $d\left(v_{4}\right)=a-2 b-1=2-b \leq 0$; If $a-b \geq 4$, then $d\left(v_{4}\right)=d\left(v_{3}\right) b(b-a+4)-b d\left(v_{3}\right)+(a-2 b-1) \leq-b d\left(v_{3}\right)+(a-2 b-1)=$ $-b\left(a b-b^{2}-2 b+1\right)+(a-2 b-1)=-a\left(b^{2}-1\right)+b^{3}+2 b^{2}-3 b-1 \leq$ $-(b+4)\left(b^{2}-1\right)+b^{3}+2 b^{2}-3 b-1=-2 b^{2}-2 b+3<0$. This is impossible.

On the other hand, it is easy to check that $G=S_{n}$ and $G=S_{\frac{n}{2}}, \frac{n}{2}$ are 2-walk $(n, 0)$-parabolic graph and $\left(\frac{n}{2}+2,1\right)$-parabolic graph, respectively.

From above, we have

Theorem 7. A tree with $n \geq 3$ vertices has exactly two main signless Laplacian eigenvalues if and only if $G$ is the star $S_{n}$ or the double star $S_{\frac{n}{2}}, \frac{n}{2}$. 
It was showed in [4] that the trees with $n \geq 3$ vertices has exactly two main eigenvalues (of adjacent matrix) are $S_{n}, S_{\frac{n}{2}, \frac{n}{2}}$ and $T_{a}$. But from Theorem 7 , we know that $T_{a}$ is not a tree with exactly two main signless Laplacian eigenvalues, where $T_{a}(a \geq 2)$ is defined in [4] to be the tree with one vertex $v$ of degree $a^{2}-a+1$ while every neighbor of $v$ has degree $a$ and all remaining vertices are pendant.

\section{Unicyclic graphs with exactly two main sign- less Laplacian eigenvalues}

In this section, we will determine all unicyclic graphs with exactly two main signless Laplacian eigenvalues.

The unique unicyclic graph with $n$ vertices and the minimum degree $\delta \geq 2$ is the cycle $C_{n}$, and it is regular. By Theorem 1 , it has exactly one main signless Laplacian eigenvalues. So, we only need to consider the unicyclic graphs with the minimum degree $\delta=1$.

Remark 1. If $G$ is a 2 -walk $(a, b)$-parabolic graph with $\delta(G)=1$, then $a-b \geq 3$ since there is a pendent vertex $x$ with the only incident edge $x y$ in $G$ and $d(y)=s(x)=-1+a-b \geq 2$.

Let $\mathscr{G}_{a, b}=\{G: G$ is a 2 -walk $(a, b)$-parabolic unicyclic graph with $\delta(G)=$ $1\}$, and for each $G \in \mathcal{G}_{a, b}$, let $G_{0}$ be the graph obtained from $G$ by deleting all pendant vertices. If $v \in V\left(G_{0}\right)$, we use $d_{G_{0}}(v)$ to denote the degree of the vertex $v$ in $G_{0}$.

Lemma 8. If $G \in \mathscr{G}_{a, b}$ and $v \in V\left(G_{0}\right)$, then $d(v)=d_{G_{0}}(v)$ or $d(v)=$ $a-b-1$.

Proof. If there is a pendant $x$ adjacent to $v$ in $G$, then $d(v)=s(x)=$ $a-b-1$ by (4). Otherwise, $d(v)=d_{G_{0}}(v)$.

Lemma 9. If $G \in \mathscr{G}_{a, b}$, then (i) $\delta\left(G_{0}\right) \geq 2$; (ii) $a-b \geq 4$ and $a \geq 5$. 
Proof. (i) If $\delta\left(G_{0}\right)=1$, then there is $y \in V\left(G_{0}\right)$ such that $d_{G_{0}}(y)=1$, and there must exist a pendant vertex $x$ adjacent to $y$ in $G$. By (44), $d(y)=$ $s(x)=-1+a-b$, this shows that there are $a-b-2$ pendant vertices adjacent to $y$ in $G$. Let $z$ be the unique non-pendant vertex adjacent to $y$ in $G$, then $s(y)=\sum_{w \in N_{G}(y)} d(w)=d(z)+(a-b-2)$. By (4), we know

$$
\begin{aligned}
s(y) & =-d^{2}(y)+a d(y)-b \\
& =-(a-b-1)^{2}+a(a-b-1)-b
\end{aligned}
$$

and $d(z)=s(y)-(a-b-2)=-(a-b-1)^{2}+a(a-b-1)-b-(a-b-2)$,

$$
\text { i.e., } \quad d(z)=a b-b^{2}-2 b+1
$$

So,

$$
d(z)-d(y)=\left(a b-b^{2}-2 b+1\right)-(a-b-1)=(b-1)(a-b-2)
$$

(I) If $b=0$, then by (7), $d(z)=1$. This is impossible since $z$ is a nonpendant vertex adjacent to $y$ in $G$.

(II) If $b=1$, then by (7) and (8), $d(z)=a-2$ and $d(y)=d(z)=a-2$.

From (4), $s(z)=-(a-2)^{2}+a(a-2)-1=2 a-5=d(y)+d(z)-1$. This shows that all the vertices adjacent to $z$, except $y$, are pendant vertices. So, $G$ is a double star with the centers $z$ and $y$. This is impossible since $G \in \mathscr{G}_{a, b}$.

(III) If $b \geq 2$, then no pendant vertex is adjacent to $z$ in $G$; Otherwise, $d(z)=s(u)=-1+a-b \geq 2$, where $u$ is a pendant vertex is adjacent to $z$. This implies that $d(z)=d(y)$ and $a-b \geq 3$, contradicting with (8). 
By (41), we have $s(z)=-d^{2}(z)+a d(z)-b$. And

$$
\begin{aligned}
s(z) & =\sum_{w \in N_{G}(z)} d(w)=\sum_{w \in N_{G_{0}}(z)} d(w) \\
& =d(y)+\sum_{w \in N_{G_{0}}(z) \backslash\{y\}} d(w) \\
& \geq d(y)+2(d(z)-1) \\
& =a-b-1+2(d(z)-1)
\end{aligned}
$$

So, $-d^{2}(z)+a d(z)-b \geq a-b-1+2(d(z)-1)$

$$
d^{2}(z)-(a-2) d(z)+a-3 \leq 0
$$

and

$$
1 \leq d(z) \leq a-3
$$

By (7),$a b-b^{2}-2 b+1 \leq a-3$, i.e., $b^{2}+(2-a) b+a-4 \geq 0$. Then

$$
b \leq \frac{(a-2)-\sqrt{(a-4)^{2}+4}}{2} \quad \text { or } \quad b \geq \frac{(a-2)+\sqrt{(a-4)^{2}+4}}{2}
$$

From Theorem $6, a^{2}>8 b \geq 16$, i.e., $a>4$, and $\frac{(a-2)-\sqrt{(a-4)^{2}+4}}{2}<$ $\frac{(a-2)-(a-4)}{2}=1$. So, $b \geq \frac{(a-2)+\sqrt{(a-4)^{2}+4}}{2}$. But

$2 \leq d(y)=a-b-1 \leq a-\frac{(a-2)+\sqrt{(a-4)^{2}+4}}{2}-1=\frac{a-\sqrt{(a-4)^{2}+4}}{2}$.

We have $a-4 \geq \sqrt{(a-4)^{2}+4}$. This is impossible.

Summarizing (I)-(III) above, we have $\delta\left(G_{0}\right) \geq 2$.

(ii) Because $G \in \mathscr{G}_{a, b}, \delta(G)=1$. There is a pendent vertex $x$ and the only edge $x y$ incident with $x$ in $G$. $d(y)=s(x)=a-b-1$ by (4). From (i), $d(y) \geq d_{G_{0}}(y)+1 \geq \delta\left(G_{0}\right)+1 \geq 3$. So, $a-b \geq 4$. 
Since

$$
\begin{aligned}
s(y) & =d(y)-d_{G_{0}}(y)+\sum_{w \in N_{G_{0}}(y)} d(w) \\
& \geq d(y)-d_{G_{0}}(y)+2 d_{G_{0}}(y) \\
& \geq a-b-1+2=a-b+1,
\end{aligned}
$$

by (3), we have

$$
\begin{aligned}
a & =\frac{s(y)-s(x)}{d(y)-d(x)}+d(y)+d(x) \\
& \geq \frac{(a-b+1)-(a-b-1)}{a-b-2}+(a-b-1)+1 \\
& =\frac{2}{a-b-2}+a-b>a-b \geq 4 .
\end{aligned}
$$

So, $a \geq 5$ since $a$ is a integer from Theorem 6 .

In the following, we determine all unicyclic graphs with exactly two main signless Laplacian eigenvalues.

Let $G_{1}$ be the unicyclic graph with $n$ vertices obtained by attaching $k \geq 1$ pendant vertices to each vertex of a cycle with length $r$, where $n=(k+1) r$. It was showed in [5] that $G_{1}$ is the only connected graph with exactly two main eigenvalue (of adjacent matrix). $G_{2}$ is the unicyclic graph with $n$ vertices obtained from the cycle $u_{1} u_{2} \cdots u_{3 t}$ by attaching one pendant vertices to the vertex $u_{3 s+1}$ for $s=0,1, \cdots, t-1$, where $n=4 t$ (see Figure 1 ).

Theorem 10. Let $G$ be a unicyclic graphs with $n$ vertices different from the cycle $C_{n}$. $G$ has exactly two main signless Laplacian eigenvalues if and only if $G$ is isomorphic to one of graphs $G_{1}$ and $G_{2}$, both given in Figure 1.

Proof. First, it is easy to check that $G_{1}$ is a 2 -walk $(k+5,2)$-parabolic graph and $G_{2}$ is a 2-walk $(5,1)$-parabolic graph.

Next, because $G \in \mathscr{G}_{a, b}$ is unicyclic, $G_{0}$ is a cycle from Lemma 9 . Let $G_{0}=C_{r}=u_{1} u_{2} \ldots u_{r} u_{1}$, then $d\left(u_{i}\right) \in\left\{d_{G_{0}}\left(u_{i}\right), a-b-1\right\}=\{2, a-b-1\}$ from Lemma 8, where $1 \leq i \leq r$. 
(i) If $d\left(u_{1}\right)=d\left(u_{2}\right)=\cdots=d\left(u_{r}\right)=a-b-1$, then $u_{i}$ has $k=a-b-3$ pendant vertices for $1 \leq i \leq r$. So, $G \cong G_{1}$.

(ii) If there is $u_{i} \in V\left(G_{0}\right)$ such that $d\left(u_{i}\right)=2$ for some $i \in\{1,2, \cdots, r\}$, then by (4), we have

$$
d\left(u_{i-1}\right)+d\left(u_{i+1}\right)=s\left(u_{i}\right)=-d^{2}\left(u_{i}\right)+a d\left(u_{i}\right)-b=-4+2 a-b
$$

Without loss of generality, we assume that $d\left(u_{i+1}\right) \geq d\left(u_{i-1}\right)$. From Lemma 9 (ii), $d\left(u_{i-1}\right)+d\left(u_{i+1}\right)=s\left(u_{i}\right)=(a-b-4)+a \geq 5$. Since $d\left(u_{i-1}\right), d\left(u_{i+1}\right) \in$ $\left\{d_{G_{0}}\left(u_{i}\right), a-b-1\right\}=\{2, a-b-1\}$, we have $d\left(u_{i+1}\right)=d\left(u_{i-1}\right)=a-b-1$, or $d\left(u_{i+1}\right)=a-b-1$ and $d\left(u_{i-1}\right)=2$.

If $d\left(u_{i+1}\right)=d\left(u_{i-1}\right)=a-b-1$, then $b=2$ by (9). From (41), $s\left(u_{i-1}\right)=$ $-(a-b-1)^{2}+a(a-b-1)-b=3 a-11$. And

$$
s\left(u_{i-1}\right)=d\left(u_{i-2}\right)+d\left(u_{i}\right)+\left(d\left(u_{i-1}\right)-d_{G_{0}}\left(u_{i-1}\right)\right)
$$

i.e., $d\left(u_{i-2}\right)=s\left(u_{i-1}\right)-d\left(u_{i}\right)-d\left(u_{i-1}\right)+d_{G_{0}}\left(u_{i-1}\right)=(3 a-11)-2-(a-b-$ 1) $+2=2 a-8$. From Lemma $8, d\left(u_{i-2}\right)=2 a-8 \in\left\{a-b-1, d_{G_{0}}\left(u_{i-2}\right)\right\}=$ $\{a-3,2\}$. We have $a=5$, and $a-b=3<4$, contradicting with Lemma 9(ii).

If $d\left(u_{i+1}\right)=a-b-1$ and $d\left(u_{i-1}\right)=2$, then $a=5$ from (91). By (4), $s\left(u_{i+1}\right)=-(a-b-1)^{2}+a(a-b-1)-b=4+2 b-b^{2}$. And

$$
s\left(u_{i+1}\right)=d\left(u_{i+2}\right)+d\left(u_{i}\right)+\left(d\left(u_{i+1}\right)-2\right)
$$

i.e., $d\left(u_{i+2}\right)=s\left(u_{i+1}\right)-d\left(u_{i}\right)-d\left(u_{i+1}\right)+2=\left(4+2 b-b^{2}\right)-2-(a-b-1)+2=$ $3 b-b^{2}$. From Lemma $8, d\left(u_{i+2}\right)=3 b-b^{2} \in\left\{a-b-1, d_{G_{0}}\left(u_{i-2}\right)\right\}=\{4-b, 2\}$. We have $b=1$ or $b=2$. And $a-b \geq 4$ from Lemma 9. So, $b=1$ and $d\left(u_{i+1}\right)=3, d\left(u_{i+2}\right)=2$.

By (4) again, 


$$
\begin{aligned}
& d\left(u_{i+3}\right)+d\left(u_{i+1}\right)=s\left(u_{i+2}\right)=-d^{2}\left(u_{i+2}\right)+5 d\left(u_{i+2}\right)-1=5, \text { and } d\left(u_{i+3}\right)= \\
& 5-d\left(u_{i+1}\right)=2 ; \\
& \quad d\left(u_{i+4}\right)+d\left(u_{i+2}\right)=s\left(u_{i+3}\right)=-d^{2}\left(u_{i+3}\right)+5 d\left(u_{i+3}\right)-1=5, \text { and } d\left(u_{i+4}\right)= \\
& 5-d\left(u_{i+2}\right)=3 ; \\
& \quad d\left(u_{i+5}\right)+d\left(u_{i+3}\right)+1=s\left(u_{i+4}\right)=-d^{2}\left(u_{i+4}\right)+5 d\left(u_{i+4}\right)-1=5, \text { and } \\
& d\left(u_{i+5}\right)=5-d\left(u_{i+3}\right)-1=2 .
\end{aligned}
$$

Continuing like this, we have

$$
d\left(u_{k}\right)= \begin{cases}2, & k-i \equiv 0,2(\bmod 3) \\ 3, & k-i \equiv 1(\bmod 3)\end{cases}
$$

So, $r \equiv 0(\bmod 3)$ and $G \cong G_{2}$.

The results on main sigenless Laplacian eigenvalues presented in Section 2 are useful to the problem of characterizing graphs with a given number of main sigenless Laplacian eigenvalues. And Theorems 7 and 10 show that the set of graphs with a given number of main sigenless Laplacian eigenvalues is not identical with the set of graphs a given number of main eigenvalues (of adjacent matrix).

\section{References}

[1] D. Cvetković, P. Rowlinson, S. Simić, Eigenspaces of Graphs, Combridge University Press, Cambridge, 1997.

[2] D. Cvetković, M. Peterić, A table of connected graphs on six vertices, Discrete Math. 50 (1984) 37-49.

[3] E. M. Hagos, Some results on graph spectra, Linear Algebra Appl. 356 (2002) 103-111.

[4] Y. Hou, H. Zhou, Trees with exactly two main eigenvalues, Acta of Hunan Normal University, 28 (2) (2005) 1-3 (in Chinese). 
[5] Y. Hou, F. Tian, Unicyclic graphs with exactly two main eigenvalues, Appl. Math. Lett. 19 (2006) 1143-1147.

[6] Z. Hu, S. Li, C. Zhu, Bicyclic graphs with exactly two main eigenvalues, Linear Algebra Appl. 431 (2009) 1848-1857.

[7] P. Rowlinson, The main eigenvalues of a graph: a survey, Applicable Analysis and Discrete Mathematics, 1 (2007) 445-471.

[8] D. Cvetković, Signless Laplacians and line graphs, Bull. Acad. Serbe Sci. Arts, Cl. Sci. Math. Natur., Sci. Math. 131(30) (2005), 85-92.

[9] D. Cvetković, P. Rowlinson, S. K. Simić, Signless Laplacians of finite graphs, Linear Algebra Appl., 423(2007), 155-171.

[10] D. Cvetković, P. Rowlinson, S. K. Simić, Eigenvalue bounds for the signless Laplacian, Publ. Inst. Math. (Beograd), 81(95)(2007), 11-27.

[11] D. Cardoso, D. Cvetković, P. Rowlinson, S. K. Simić, A sharp lower bound for the least eigenvalue of the signless Laplacian of a non-bipartite graph, Linear Algebra Appl., 429(2008), 2770-2780.

[12] D. Cvetković, New theorems for signless Laplacian eigenvalues, Bull. Acad. Serbe Sci. Arts, Cl. Sci. Math. Natur., Sci. Math. 137(33) (2008), 131-146.

[13] K. Feng, Algebraic Number Theory, Beijing, Science Press, 2000 (in Chinese). 